\title{
Stability of Packed Bed Thermoclines
}

\author{
Tristan R.G. Davenne*, Seamus D. Garvey, Bruno Cardenas, James P. Rouse \\ Faculty of Engineering, University of Nottingham, University Park, Nottingham, NG7 2RD, UK
}

\begin{abstract}
Packed bed thermoclines have attracted considerable interest as an economical method for storing large amounts of thermal energy. They are a constituent part of a range of proposed thermo-mechanical energy storage systems, such as Adiabatic Compressed Air Energy Storage (ACAES) and Pumped Thermal Energy Storage (PTES). The low cost of the thermal storage media (crushed rock or gravel) means that even with the cost of the required compression and expansion equipment, these systems potentially have a lower Levelised Cost of Storage than batteries, especially for grid scale storage. Packed bed thermoclines rely on a stratified temperature gradient (thermal front) between heated material at the top and cooler material at the bottom. The stability of this thermal front can affect the exergetic efficiency of the store. We present a simple criterion for the stability of a thermal front and show that during discharge of a hot store, a small cold region in the thermal front can develop into a cold tunnel that propagates ahead of the main thermal front. By contrast, the presence of a small hot region at the thermal front prior to charging with hot gas is shown to be quickly dissipated. We also calculate a theoretical critical perturbation size required for a cold tunnel to develop ahead of the thermal front. Below this size transyerse thermal diffusion is able to dissipate perturbations before they can develop. Three dimensional Computational Fluid Dynamics simulations are used to accurately visualise thermal front instabilities and also to quantify their effect on the exergetic efficiency of a cycling thermal store. Adding a small high void fraction region near the bottom of the thermal store caused a significant disruption of the thermal front on each discharge cycle and resulted in a $4.5 \%$ increase in the exergy loss rate. Low void fraction adjacent to the walls of the thermal store, which typically occurs during packing, caused a more significant $63 \%$ increase in the exergy loss rate relative to a uniformly packed thermal store.
\end{abstract}

\section{Introduction}

The storage of thermal energy in packed beds of rock has been the subject of numerous studies in recent years. This interest has been fuelled by new proposals to store energy $[1,2,3,4,5,6,7]$ including adiabatic compressed air energy storage (ACAES) and pumped thermal energy storage (PTES), which all depend on exergetically efficient thermal stores. A stratified packed bed thermal store is charged with hot gas entering at the top, then giving up its heat to the rocks as it flows down through the bed. Discharge is affected by pumping cold gas into the bottom, which is heated by the hot rock before leaving at the top of the store. A thermal front (also known as a thermocline) moves down and up the packed bed as it is charged and discharged. The behaviour and performance of packed bed thermal stores is often simulated with one dimensional models which assume uniformity in the horizontal direction perpendicular to the main vertical flow through the thermal store $[8,9,10,11,12,13]$. While these may give reasonable predictions of thermal store performance, they clearly cannot predict any 3D phenomena within the packed bed.

There are experimentally validated 3D CFD simulations of packed bed thermal stores reported in the literature that do consider transverse temperature variations in thermal stores. For example, Cascetta et al. [14] show that a radial distribution in void fraction causes non-uniformities in the thermocline. Bruch et al. [15] note that stable thermocline behaviour can be degraded by non-uniformities in the inlet flow. They evaluate a thermal store where the bottom port is on one side of the store and for certain flow regimes this asymmetry causes significant disruption of the thermal front. Zavatonni et al. [16] obtained a good match between their CFD predictions and their experimental setup by using a non-uniform porosity model. These papers constitute good evidence that the stability of the thermal front is sensitive to variations in porosity. Qin et al. [17] study the stability of a molten salt thermocline packed with a quartz or sand filler. They deduced a critical flow velocity that must not be exceeded to avoid the onset of an instability in the thermocline, described as viscous fingering. The addition of quartz or sand filler materials was found to improve stability as compared to a simple liquid thermocline. The physics of stability of a packed bed that uses liquid as the heat transfer media is quite different to one that uses gas. A liquid can generally be considered as incompressible and the pressure drop required to push the liquid through the packed bed will tend to reduce with increased temperature due to reducing viscosity. With a gas, pressure drop will increase with increasing temperature, primarily due to the

*corresponding author, tristan.davenne@stfc.ac.uk 
relationship between density and temperature for a perfect gas. The fundamental difference between a gaseous and liquid heat transfer media is well illustrated by considering the pressure drop in a pipe using the Darcy-Weisbach equation [18]. For illustrative purposes we consider that for laminar flow the pressure drop per unit length is proportional to kinematic viscosity and can be written as follows

$$
\frac{\Delta P}{L}=\frac{128 m v}{\pi \beta^{4}} \quad 1
$$

where $\beta$ is particle diameter, $\dot{m}$ is mass flow rate and $v$ is the kinematic viscosity of the fluid. Simply plotting kinematic viscosity which is a fluid property as a function of temperature shows how the pressure drop will vary with temperature for a given mass flow of each fluid (Figure 1).

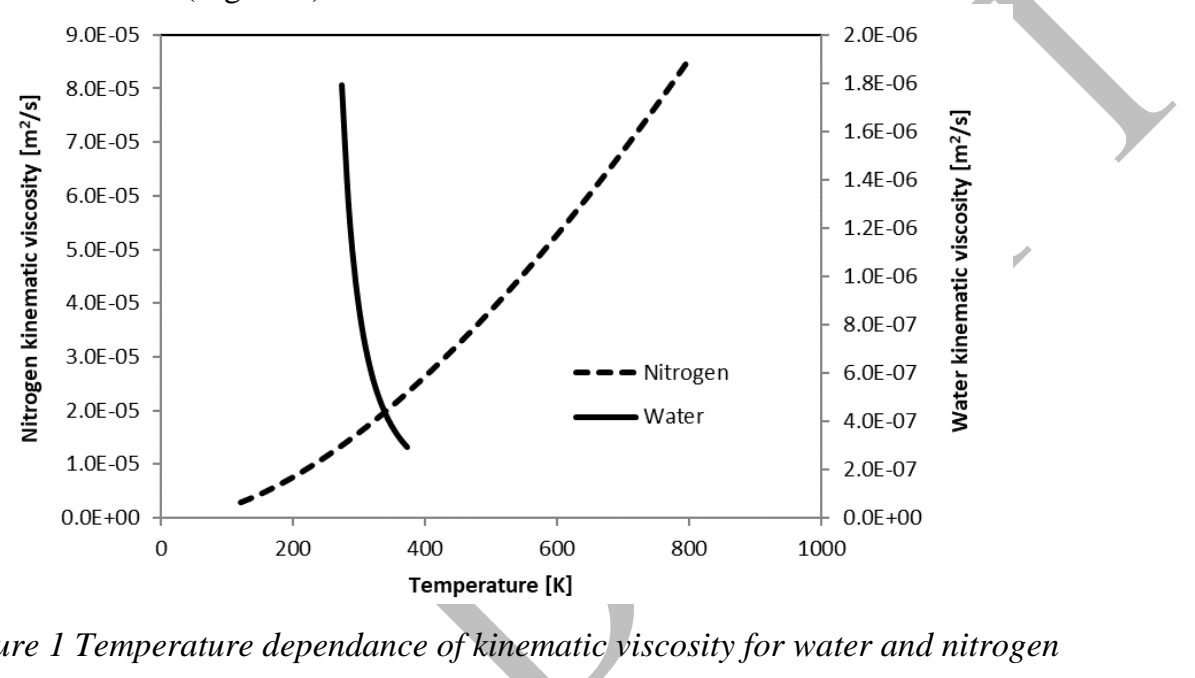

The thermocline, in a purely liquid thermal store with no packing material, is prone to a whole set of instabilities that are not present in the context of a packed bed where the scale of turbulence and large-scale fluid structures is limited by the length scale of the packing material. For example, Van-Berkel et al. [19] made a comprehensive study of the stability of liquid thermoclines showing the development of Kelvin-Helmholtz like waves in the thermal front, and Tinaikar et al. [20] studied the widening of the thermocline due to vortices generated in this region caused by the momentum of the inlet flow. Returning to packed beds with gas as the heat transfer fluid, Zavatonni et al. [21] also studied a novel method of using phase change materials in combination with a sensible heat packed bed to improve thermal store performance. They consider the result of non-uniform porosity on the thermal front, referred to here as channelling, and then show that the addition of phase change material at the top of the thermal store helps to keep a stable discharge temperature in spite of their disrupted thermal front. A common theme in the reported experimental results and CFD analysis of packed bed thermal stores is the presence of transverse variations in the thermal profile and this is often shown to be due to non-uniform void fraction or non-uniformities in the flow.

In this paper, we focus on thermal stores with a perfect gas as the heat transfer medium. We make the fundamental observation that thermoclines are stable during the charging phase but inherently unstable during discharge. While evidence of this instability can be seen in some of the results presented in the literature, we believe this observation is a point of academic interest and may be useful information for the designers of thermal stores who are striving for maximum possible efficiency. We present a theoretical model of a simplified packed bed which highlights that when cooling a packed bed, any preferentially cooled areas will result in a lower pressure drop leading to more flow and further cooling in a positive feedback loop. A small perturbation in the thermal front is seen to grow into a cold tunnel, which leads the main thermal front. When heating a packed bed, any preferentially heated area in the thermal front will have increased pressure drop, which limits flow and results in a damping effect where perturbations in the thermal front tend to be smoothed out. We also consider the effect of transverse thermal diffusion on any instabilities and calculate a theoretical critical perturbation size. Above the critical size, perturbations can develop into significant disruptions of the thermal front but below the critical size transverse diffusion is dominant and acts to dissipate the perturbation. Finally, we present results of CFD simulations of an example packed bed thermal store. We calculate 
the exergy loss due to the development of a thermal front following a repeated charge/discharge cycle. We compare the exergy loss rate for the thermal store with a perfect uniform void fraction to three test cases with different nonuniformities in the void fraction.

\section{Thermal Store Stability Criterion}

It is easy to imagine that small non-uniformities in the radial/transverse temperature profile of a packed bed could arise due to non-uniformities in the void fraction or imperfect inlet manifolds. The aim of this section of the paper is to establish a criterion to determine if these non-uniformities in the thermal front can grow or not. We start with the pressure drop across a packed bed which can be calculated from the Ergun equation [22] as follows:

$$
\frac{\Delta P}{L}=a M+b M^{2}
$$

where $\mathrm{L}$ is the length of the packed bed, $\mathrm{M}$ is the mass flow per unit area flowing through the packed bed, and $\mathrm{A}$ and $\mathrm{B}$ are temperature dependant and defined as follows:

$$
a=\frac{150 \mu(1-\varepsilon)^{2}}{\rho d^{2} \varepsilon^{3}} \quad b=\frac{1.75 \rho(1-\varepsilon)}{\rho^{2} d \varepsilon^{3}}
$$

Consider the pressure drop across a relatively small column shaped control volume within a packed bed thermal store (Figure 2).

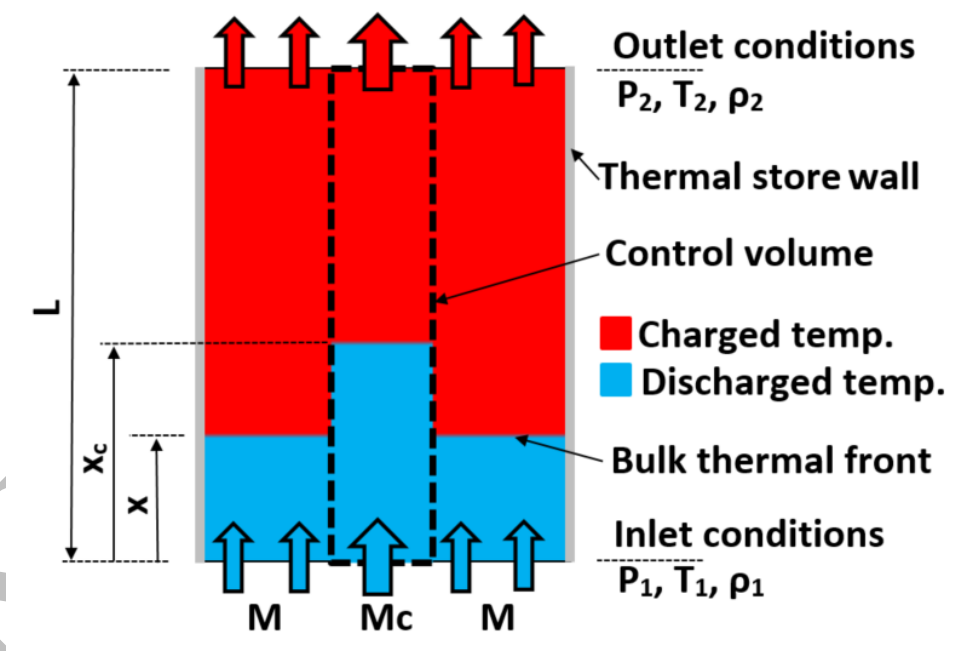

Figure 2 Schematic of thermal store showing control volume for stability analysis

The mass flow rate through the entire store is equal to the sum of the mass flow through the control volume and the rest of the packed bed i.e.

$$
M_{T}\left(S+S_{c}\right)=M S+M_{c} S_{c} \quad 4
$$

The portion of flow that travels through each of the two possible paths depends on the resistance to flow in that path and we assume that there is no heat or mass transfer through the vertical sides of the control volume. If the packed bed is partially charged, and assuming a sharp edged ideal thermal front, we can approximate the pressure drop across the bed as being made up of two components, the first component due to a length, $\mathrm{x}$, at the inlet temperature and the second component due to a length, L-X, at the outlet temperature. The pressure drop across the control volume is written as 


$$
\frac{\Delta P}{L}=\left(a_{1} M_{c}+b_{1} M_{c}^{2}\right) R_{c}+\left(a_{2} M_{c}+b_{2} M_{c}^{2}\right)\left(1-R_{c}\right)
$$

where $R_{c}=\frac{x_{c}}{L}$ represents the fill ratio in the control volume and subscripts 1 and 2 refer to inlet and outlet conditions respectively. Rearranging for $R_{c}$ gives

$$
R_{c}=\frac{\frac{\Delta P}{L}-\left(a_{2} M_{c}+b_{2} M_{c}^{2}\right)}{\left(a_{1} M_{c}+b_{1} M_{c}^{2}-a_{2} M_{c}-b_{2} M_{c}^{2}\right)}
$$

Now differentiating with respect to $M_{c}$ using the quotient rule we have

$$
\frac{\partial R_{c}}{\partial M_{c}}=\frac{g^{\prime}\left(M_{c}\right) h\left(M_{c}\right)-g\left(M_{c}\right) h^{\prime}\left(M_{c}\right)}{h\left(M_{c}\right)^{2}}
$$

Where

and

$$
\begin{gathered}
g\left(M_{c}\right)=\frac{\Delta P}{L}-\left(a_{2} M_{c}+b_{2} M_{c}^{2}\right) \\
h\left(M_{c}\right)=\left(a_{1} M_{c}+b_{1} M_{c}^{2}-a_{2} M_{c}-b_{2} M_{c}^{2}\right)
\end{gathered}
$$

$$
\begin{gathered}
g^{\prime}\left(M_{c}\right)=-a_{2}-2 b_{2} M_{c} \\
h^{\prime}\left(M_{c}\right)=\left(a_{1}+2 b_{1} M_{c}-a_{2}-2 b_{2} M_{c}\right)
\end{gathered}
$$

writing out in full and noting that

gives

$$
\frac{\partial M_{c}}{\partial R_{c}}=1 /\left(\frac{\partial R_{c}}{\partial M_{c}}\right)
$$

$$
\frac{\partial M_{c}}{\partial R_{c}}=\frac{\left(a_{1} M_{c}+b_{1} M_{c}^{2}-a_{2} M_{c}-b_{2} M_{c}^{2}\right)^{2}}{\frac{\Delta P}{L}\left(a_{2}-a_{1}+2 M_{c}\left(b_{2}-b_{1}\right)\right)+M_{c}^{2}\left(a_{2} b_{1}-a_{1} b_{2}\right)}
$$

If $\frac{\partial M_{c}}{\partial R_{c}}>0$ or simply

$$
\frac{\Delta P}{L}\left(a_{2}-a_{1}+2 M_{c}\left(b_{2}-b_{1}\right)\right)+M_{c}^{2}\left(a_{2} b_{1}-a_{1} b_{2}\right)>0
$$

this signifies that a small increase in fill ratio within the control volume will cause the mass flow in the control volume to increase. This highlights the presence of a positive feedback instability where an increasing proportion of the total mass flow passes through the control volume giving rise to a cold tunnelling effect where the control volume fill ratio moves further and further ahead of the bulk fill ratio. The condition in equation 14 is met when a hot thermal store is being discharged by a flow of cold gas (as shown in Figure 3), or when a discharged low temperature store is being charged by a very cold gas. 


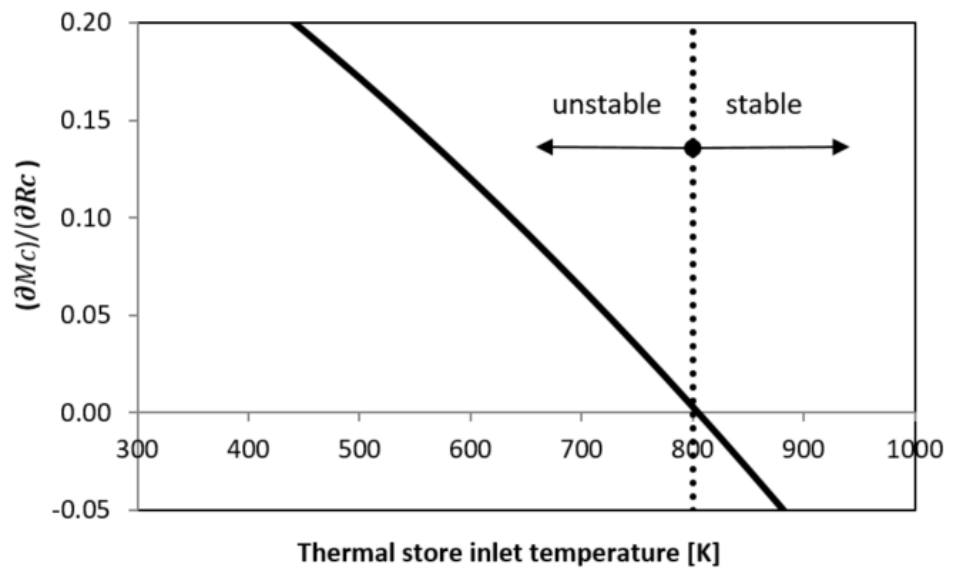

Figure 3 Stability criteria plotted for example packed bed thermal store with nitrogen gas as the heat transfer media. Plot shows stability as a function of inlet temperature for a store with an outlet temperature of $800 \mathrm{~K}$ (i.e. $T_{1}$ is the $x$ axis \& $T_{2}=800 \mathrm{~K}$ )

\section{Critical perturbation size}

The growth of a perturbation during discharge of a hot store or charging of a cold store depends on the strength of the instability and also the amount of transverse thermal diffusion perpendicular to the main direction of flow through the packed bed. Below a certain critical perturbation size energy flow into the perturbation to advance it ahead of the bulk thermal front will be smaller than the rate of transverse diffusion and so the perturbation will damp out. However above the critical size and energy lost by transverse diffusion will be small compared to the energy flow into the perturbation and so the perturbation will grow.

We now use a simple analysis to evaluate the critical perturbation size as a function of parameters of interest such as mass flow rate, packed bed aspect ratio, rock material thermal conductivity and diameter. First consider that the packed bed control volume has a perturbation within it of height and diameter, D (Figure 4).

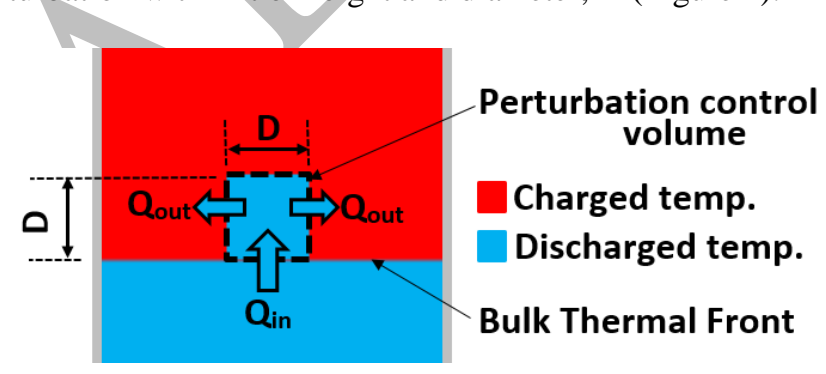

Figure 4 Section of the packed bed showing a control volume surrounding an idealised perturbation in the thermal front

We can then determine the mass flow and thus energy flow into the perturbation as a function of its size using

$$
R=x / L \text { and } R_{c}=(x+D) / L
$$

Assuming that the pressure difference across the control volume is always equal to the pressure difference across the rest of the thermal store then

$$
\frac{\left(P_{2}-P_{1}\right)}{L}=\left(A_{1} M+B_{1} M^{2}\right) R+\left(A_{2} M+B_{2} M^{2}\right)(1-R)=\left(A_{1} M_{c}+B_{1} M_{c}^{2}\right) R_{c}+\left(A_{2} M_{c}+B_{2} M_{c}^{2}\right)\left(1-R_{c}\right) 15
$$


Solving equation 15 yields the mass flow per unit area in the bulk thermal store and in the control volume as follows

with

$$
M=\frac{-F+\sqrt{F^{2}-4 E G}}{2 E} \quad 16
$$

$$
\begin{gathered}
E=\left(B_{1} R-B_{2} R+B_{2}\right)-\left(B_{1} R_{c}-B_{2} R_{c}+B_{2}\right) \frac{S^{2}}{S_{c}^{2}} \\
F=\left(A_{1} R-A_{2} R+A_{2}\right)-\left(-A_{1} R_{c}+A_{2} R_{c}-A_{2}\right) \frac{S}{S_{c}}+\frac{2 M_{T}\left(S+S_{c}\right) S}{S_{c}^{2}}\left(B_{1} R_{c}-B_{2} R_{c}+B_{2}\right) \\
G=-\frac{M_{T}\left(S+S_{c}\right)}{S_{c}}\left(A_{1} R_{c}-A_{2} R_{c}+A_{2}\right)-\frac{M_{T}{ }^{2}\left(S+S_{c}\right)^{2}}{S_{c}{ }^{2}}\left(B_{1} R_{c}-B_{2} R_{c}+B_{2}\right)
\end{gathered}
$$

and then$$
M_{c}=\frac{M_{T}\left(S+S_{c}\right)-M S}{S_{c}}
$$

It has been shown previously [14] that the thermal front velocity is proportional to mass flow rate as follows

$$
c=\frac{C p_{f}}{\rho_{s} C p_{s}(1-\varepsilon)} M
$$

This is equally valid for the column control volume

$$
c_{c}=\frac{C p_{f}}{\rho_{s} C p_{s}(1-\varepsilon)} M_{c}
$$

We can then define a slip velocity which represents the relative speed of the perturbation as compared to the bulk thermal front.

$$
c_{\text {slip }}=c_{c}-c
$$

We then assert that the energy transfer rate feeding the perturbation instability, is proportional to the previously calculated slip velocity and can be determined as follows

$$
Q_{\text {in }}=c_{\text {slip }} \pi D^{2} \rho_{s} C p_{s}(1-\varepsilon) \Delta T \quad 24
$$

This simple model assumes no heat transfers across the walls of the column shaped control volume. However we know that transverse or radial thermal dissipation will act to damp out instabilities via thermal conduction and turbulence in the packed bed. To compare the relative magnitude of energy flow in and out of the perturbation we consider a control volume around the perturbation as depicted in Figure 4. According to Ming et al. [23] the convectively enhanced transverse fluid conductivity is proportional to the static gas thermal conductivity and the Peclet number as follows

where the Peclet number is given by

$$
k_{c}=0.04 k_{f} \frac{(1-\varepsilon)}{\varepsilon} P e
$$

$$
P e=\frac{V d}{\alpha} \quad 26
$$

To obtain the effective transverse thermal conductivity of the packed bed the conductivity of the rock material must also be accounted for. Dietz [24] presented an experimentally validated relationship for the effective thermal 
conductivity of a packed bed as a function of the solid and fluid thermal conductivities. Fitting a curve to the presented data gives us the following expression for the effective transverse thermal conductivity

$$
k_{t}=k_{c}\left(1+0.85\left(\frac{k_{s}}{k_{f}}\right)^{0.09} \ln \left(\frac{k_{s}}{k_{f}}\right)\right)
$$

We then assume that across the diameter of the control volume the perturbation can be described by the following temperature profile

$$
T(r)=\frac{\Delta T}{2}\left(1+n \cos \left(\frac{\pi r}{D}\right)\right)
$$

Where $n=-1$ for cooling and $n=+1$ for heating of the packed bed. The cold flowing radially out of the cylindrical perturbation of diameter and height $\mathrm{D}$ is calculated based on the effective transverse conductivity as follows

$$
Q_{\text {out }}=k_{t} \pi D^{2} \frac{\partial T}{\partial r}\left(r=\frac{D}{2}\right)=-\frac{k_{t} D \pi^{2} \Delta T}{4} \quad 29
$$

If $\frac{Q_{\text {in }}}{Q_{\text {out }}}>1$ then the perturbation will develop and grow whereas if $\frac{Q_{\text {in }}}{Q_{\text {out }}}<1$ then transverse diffusion dominates and the perturbation will not grow. The critical perturbation size above which growth of a cold tunnel will occur can be determined from finding the value of $\mathrm{D}$ that gives rise to the following equality

$$
Q_{\text {in }}=Q_{\text {out }}
$$

We now use these expressions 24,29 and 30 to evaluate how the critical perturbation size depends on parameters of interest such as rock diameter and thermal conductivity, mass flow rate per unit area and aspect ratio. We refer to recent work by Cardenas et al. [25] on the optimum aspect ratio and rock size in packed beds to define an example packed bed thermal store for the purpose of the following calculations. The example store has a volume of $12 \mathrm{~m}^{3}$ with a cross sectional area of $4 \mathrm{~m}^{2}$ and a height of $3 \mathrm{~m}$ (Aspect ratio of 1.5) and is packed with $4 \mathrm{~mm}$ diameter spherical rocks with a thermal conductivity of $1 \mathrm{~W} / \mathrm{mK}$ and a temperature dependant specific heat capacity as shown in Figure 9. The packed bed is assumed to have a void fraction of 0.5. The heat transfer medium is taken as nitrogen which is treated as a perfect gas with a rated mass flow of $7 \mathrm{~kg} / \mathrm{s}$ which corresponds to a rated exergy flow of $0.19 \mathrm{MW}$. When all the packed rock is heated to $527^{\circ} \mathrm{C}$ (i.e. fully charged) the packed bed contains $2.3 \mathrm{GJ}$ of exergy. We then consider the effect of adjusting these main design parameters in turn. The parameter space considered covers the typical

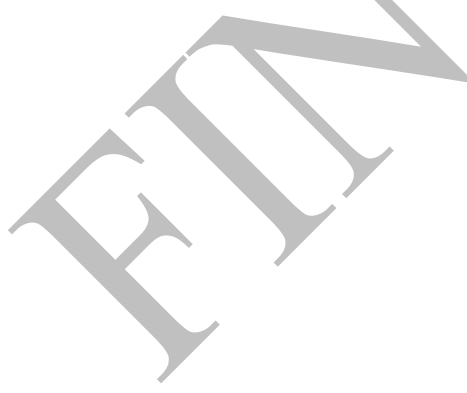


Reynolds number range experienced $(50<\operatorname{Re}<1000)$ in packed bed thermal stores. The following plots show the resulting critical perturbation size for the range of parameters considered, (Figure 5 to Figure 8).

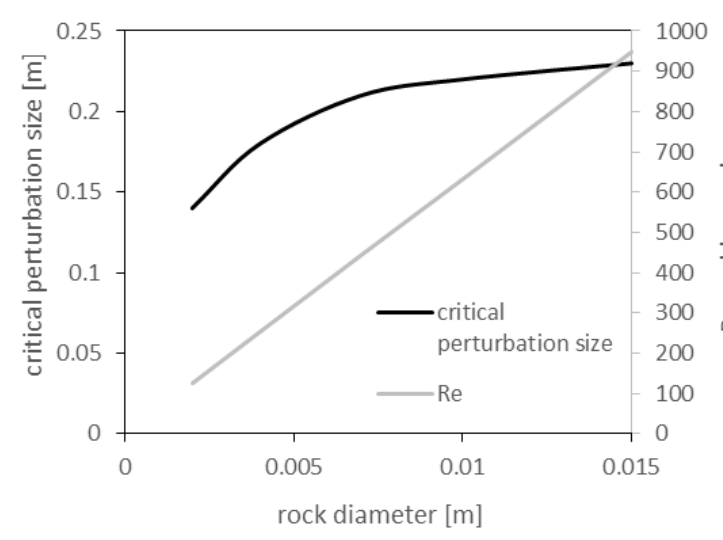

Figure 5 Critical Perturbation size as a function of rock diameter; rock thermal conductivity $=1 \mathrm{~W} / \mathrm{mK}$, aspect ratio $=0.5$, mass flow $=7 \mathrm{~kg} / \mathrm{sm}^{2}$

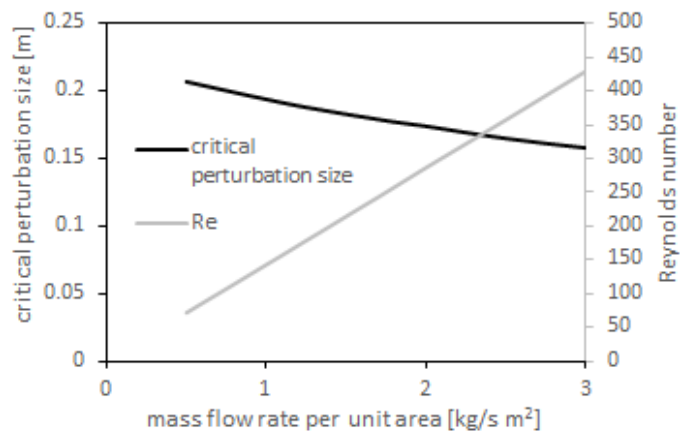

Figure 7 Critical Perturbation size as a function of mass flow through thermal store; rock diameter $=4$ $\mathrm{mm}$, aspect ratio $=0.5$, thermal conductivity $=1 \mathrm{~W} / \mathrm{mK}$

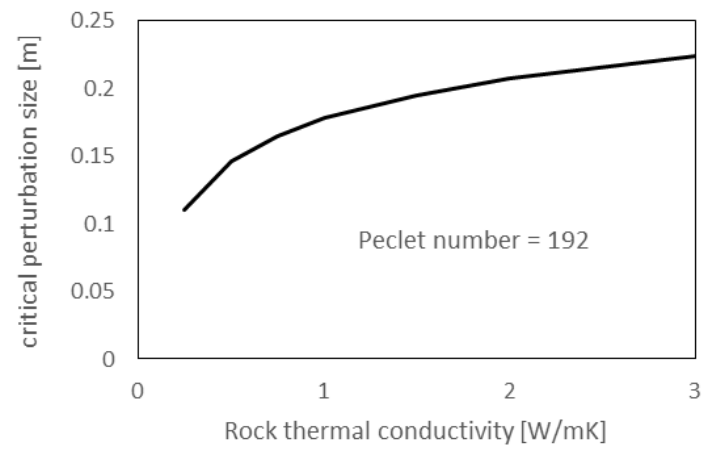

Figure 6 Critical Perturbation size as a function of rock thermal conductivity; rock diameter $=4 \mathrm{~mm}$, aspect ratio $=0.5$, mass flow $=7 \mathrm{~kg} / \mathrm{sm}^{2}$

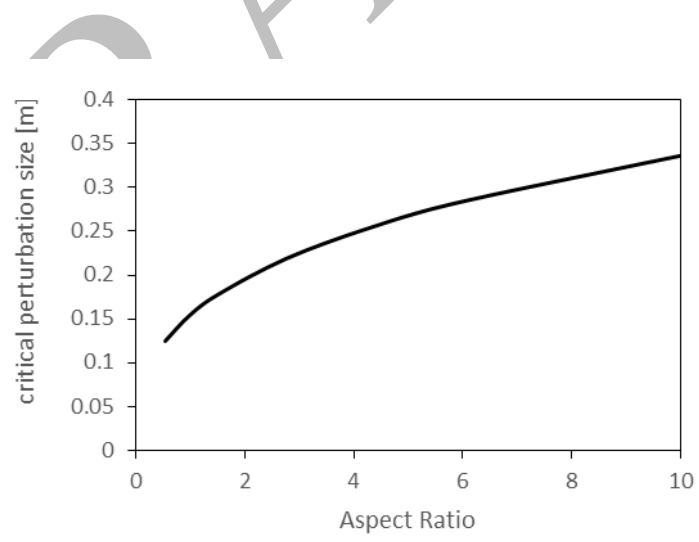

Figure 8 Critical Perturbation size as a function of aspect ratio (thermal store volume $=12 \mathrm{~m}^{3}$ ), rock diameter $=4 \mathrm{~mm}$, mass flow $=7 \mathrm{~kg} / \mathrm{sm}^{2}$, thermal conductivity $=1 \mathrm{~W} / \mathrm{mK}$

The smaller the critical perturbation size the more plausible a growing instability becomes. Smaller rocks and higher mass flows both lead to a smaller perturbation size as they both result in higher pressure drop per unit length and so increase the propensity for the flow to move to low void fraction regions. Lower rock thermal conductivity which can result from rock porosity results in reduced transverse thermal diffusion and so reduces the critical perturbation size and likelihood of propagating instabilities. Low aspect ratio results in a smaller critical perturbation size due to a given perturbation size being a larger fraction of the length of the packed bed. Varying one parameter at a time within the parameter space considered, shows that the critical perturbation size varies between 0.1 and $0.35 \mathrm{~m}$. By varying all four parameters together a minimum critical perturbation size of $0.06 \mathrm{~m}$ was found. This was found with an aspect ratio of 0.5 , mass flow rate of $12 \mathrm{~kg} / \mathrm{sm}^{2}$, rock diameter of $2 \mathrm{~mm}$ and rock thermal conductivity of $0.25 \mathrm{~W} / \mathrm{mK}$.

\section{CFD Analysis}

The idealised analysis presented above serves to indicate the conditions required for unstable growth of thermal front deformations. However, the authors believe it is useful at this point to offer results from a Computational Fluid Dynamics analysis of a three-dimensional thermal store, as this gives a more realistic and representative view of the propagation of unstable thermal fronts in particular accounting for the diffusion of the thermal front that is constantly 
taking place. We solve the Navier Stokes equations in a porous media using the CFX code [26]. The continuity and momentum equations are represented as follows

$$
\begin{gathered}
\frac{\partial \rho \varepsilon}{\partial t}+\nabla \cdot(\rho K \cdot U)=0 \\
\frac{\partial \rho \varepsilon U}{\partial t}+\nabla \cdot(\rho(K \cdot U) \mathrm{x} U)-\nabla \cdot\left(\mu K \cdot\left(\nabla U+(\nabla U)^{T}\right)\right)=-\varepsilon \sigma \cdot U-\varepsilon \nabla p
\end{gathered}
$$

Where $U$ is the vector of true velocity, $K$ is an area porosity tensor representing the area available for flow, $\mu$ is the viscosity - either laminar or effective turbulent, and $\sigma$ is the tensor representing the resistance to flow in porous media and acts in an additional source term in the momentum equation. The energy equation can be represented as follows with $\gamma$ being thermal diffusivity (either laminar or effective turbulent value is used) and $\Gamma$ represents a source or sink of energy from the solid material to the fluid.

$$
\frac{\partial \rho \varepsilon H}{\partial t}+\nabla \cdot \rho(K \cdot U H)-\nabla \cdot(\gamma K \cdot \nabla H)=\varepsilon \Gamma
$$

We simulate our example packed bed thermal store which has a cross sectional area of $4 \mathrm{~m}^{2}$ and a height of $3 \mathrm{~m}$ and is packed with $4 \mathrm{~mm}$ diameter spherical rocks with thermal conductivity of $1 \mathrm{~W} / \mathrm{mK}$. The heat transfer medium is nitrogen which is treated as a perfect gas. Typical thermal properties for quartz are assumed for the rock and this includes temperature dependant heat capacity. During the transient CFD simulations we also calculate and record the exergy within the thermal store rock and gas and the flow of exergy in and out of the store during charge and discharge phases. We use equation 34 to find the exergy value [27] associated with changing a given mass of fluid or rock from the reference temperature to a temperature at state 1 .

$$
B_{01}=m \int_{0}^{1} C_{p}\left(1-\frac{T_{0}}{T}\right) d T \quad 34
$$

The heat capacity of the rock varies increases with temperature across the temperature range of interest as shown by Hartlieb et al, [28]. This means that the cooler the rock gets the less heat that must be removed to cool it further and hence this physical property of rock encourages discharge instabilities. We use a linear approximation to account for this variation in the CFD simulations, i.e. $C p_{s}=e T+f$ as shown in Figure 9. The heat capacity of the nitrogen also increases with temperature but by less than 10\% across the temperature range of interest and as the heat capacity of the gas is small compared to the rock this is less important although it is accounted for in the simulations.

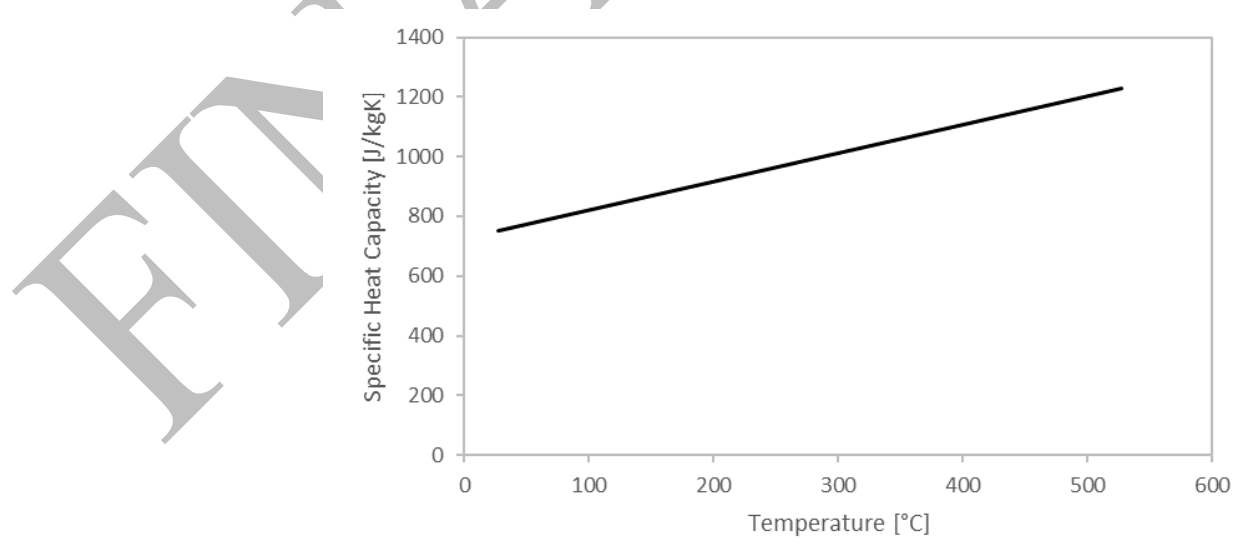

Figure 9 Dependence of rock specific heat capacity on temperature 
The exergy contained within the packed rock, when heated from the reference state 0 to state 1 can be calculated by completing the integration of equation 34 and then integrating over the volume of the packed bed as follows where $d v$ is an elemental volume within the thermal store

$$
B_{s}=\rho_{s} \int \varepsilon\left(\frac{e}{2}\left(T_{1}{ }^{2}-T_{0}{ }^{2}\right)+\left(f-e T_{0}\right)\left(T_{1}-T_{0}\right)-f T_{0} \ln \left(\frac{T_{1}}{T_{0}}\right)\right) d v
$$

The exergy contained within the gas in the store can be calculated in a similar manor. The instantaneous exergy flow rate in or out of the top of the store is obtained as

$$
B_{\text {top }}=\int\left(\int_{0}^{1}(1-\varepsilon) M C_{p f}\left(1-\frac{T_{0}}{T}\right) d T\right) d a
$$

where $d a$ is an elemental area of the top opening of the thermal store and the outside integral is over the total area of top opening. The cumulative exergy loss after operation of the thermal store for a time, $\tau$, is then calculated as follows

$$
B_{\text {loss }}(t=\tau)=\int_{0}^{\tau} B_{\text {top }} d t-B_{s}(\tau)-B_{f}(\tau)
$$

Where $B_{s}$ and $B_{f}$ represent the exergy stored in the packed rock and the fluid contained within the thermal store respectively. $B_{\text {out }}$ is the exergy flow rate out of the top of the thermal store and is positive when exergy is flowing into the store and negative when exergy flows out of the store. Any exergy that leaves the packed bed from the bottom of the store during charging is considered to be lost.

In the first transient simulation the packed bed is assumed to have a uniform packing fraction of 0.5 meaning a uniform void fraction of 0.5 . The initial condition is a fully charged store, i.e. all rock and gas in the store is at $527^{\circ} \mathrm{C} \mathrm{except}$ for a small cold region at the bottom inlet. Cold nitrogen at $27^{\circ} \mathrm{C}$ is flowed uniformly into the bottom of the thermal store to almost entirely discharge the store. Figure 10 shows the progression of the thermal front during discharge and it can be seen that the cold region at the inlet causes a significant growing disruption in the thermal front. In the second case the initial condition is a fully discharged store except for a small hot region at the top inlet. Hot gas at $527^{\circ} \mathrm{C}$ is then flowed uniformly into the top of the thermal store to almost entirely charge the store. Figure 11 shows how the hot region does not result in any transverse deformation of the thermal front and in fact is dissipated very quickly. These first two transient CFD simulations serve to validate the theoretical point that when cooling a thermal store, the thermal front is inherently unstable but when heating a store, the thermal front is stable with non-uniformities rapidly dissipated.

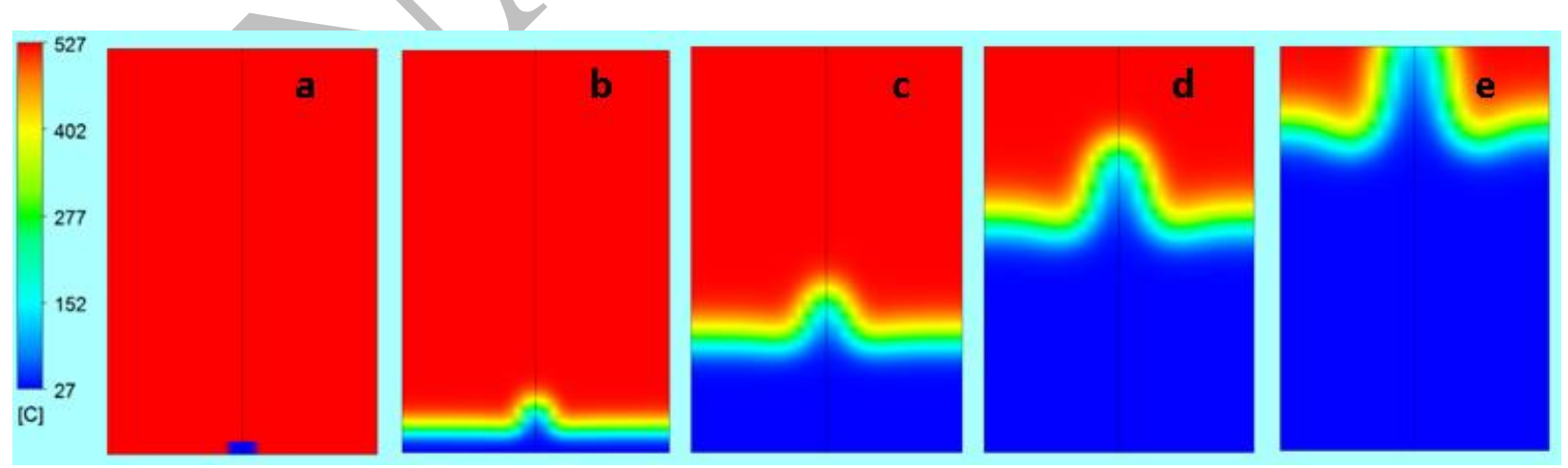

Figure 10 Cold region at the inlet of uniformly packed thermal store develops into a significant perturbation of the thermal front during discharge; each frame shows a contour plot of rock temperature on a plane in the centre of the store at different times after the discharge begins, $a=0 \mathrm{~s}, b=700 \mathrm{~s}, c=2100 \mathrm{~s}, d=5250 \mathrm{~s}, e=9000 \mathrm{~s}$ 

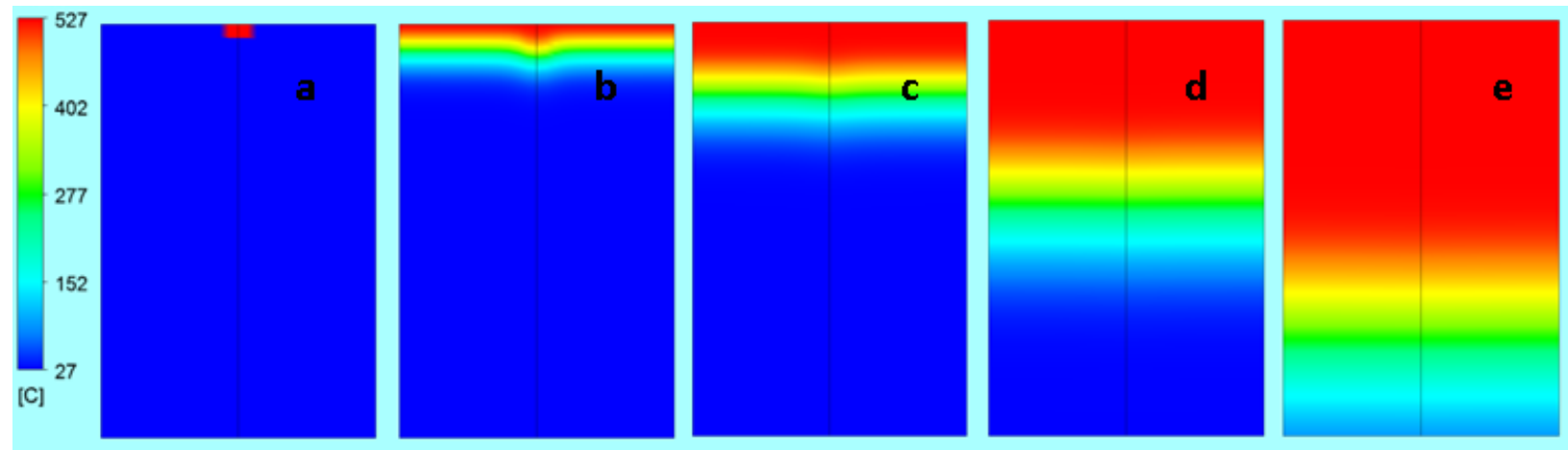

Figure 11 Hot region at the inlet of uniformly packed thermal store is dissipated during charging; each frame shows a contour plot of rock temperature on a plane in the centre of the store at different times after the charging begins, $a=0 s, b=700 s, c=2100 s, d=5250 s, e=9000 s$

We now consider how small regions of increased void fraction can affect the exergetic efficiency of the thermal store as it is cyclically charged and discharged. We consider four cases with differing small variations in void fraction as described below. Casses 1,2 and 4 are shown in Figure 12.

Case 1: uniform void fraction $=0.5$

Case 2: uniform void fraction $=0.5$ except for a region $($ size $=0.3 \mathrm{~m})$ near the base and at the centre of the packed bed with void fraction $=0.75$

Case 3 uniform void fraction $=0.5$ except for five regions ( size $=0.3 \mathrm{~m}$ ) equally spread around the base of the thermal store with void fraction $=0.75$

Case 4 uniform void fraction $=0.5$ except for a $2 \mathrm{~cm}$ thick region adjacent to the walls of the thermal store with void fraction $=0.75$
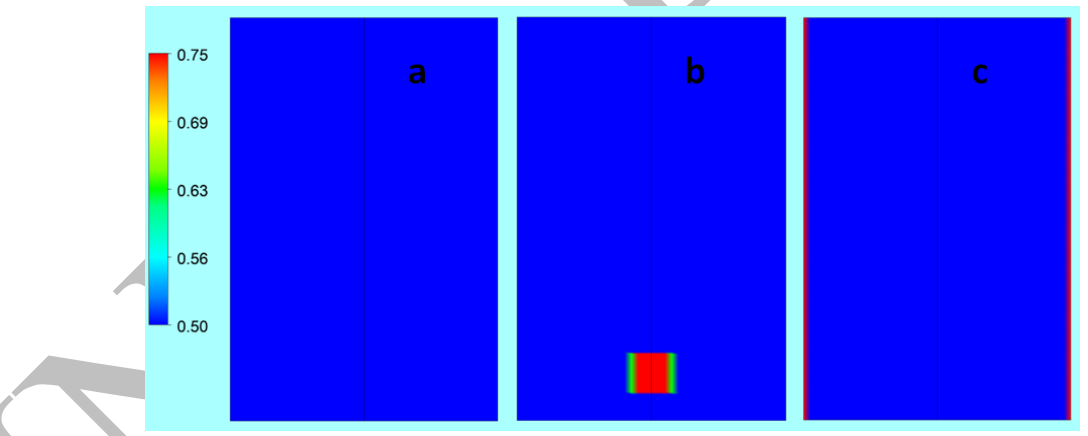

Figure 12 Void fraction on a plane through centre of thermal store; $a=c a s e 1 ; b=c a s e 2 ; c=c a s e 4$

We apply a simple charge discharge cycle (Figure 17a) to each thermal store which includes 5 repetitive cycles as by that point the behaviour of the thermal store becomes repetitive. Figure 13 shows the progression of the thermal front during the fifth charge/discharge cycle for the case of a uniform void fraction of 0.5. It can be seen that the front is steeper on discharge than on charging; this is due to the dependence of the thermal front velocity on temperature [29]. There is no transverse disruption of the thermal front as expected, however exergy is being lost due to the smearing of the thermal front and the rate of exergy loss is plotted in Figure 17b. Over the first five cycles the average exergy loss rate is calculated as $2.9 \mathrm{~kW}$, which is $1.5 \%$ of the rated exergy flow rate.

We now consider the same charge/discharge cycle applied to the thermal store with a locally increased void fraction in a $0.3 \mathrm{~m}$ sized central region near the base of the store, i.e. case 2 . At the start of every discharge cycle, the high void fraction region is seen to trigger the formation of a cold tunnel propagating ahead of the thermal front (Figure 14 \& Figure 15). During the charging phase some smoothing of the disrupted thermal front is evident. This behaviour is 
observed on each charge/discharge cycle and leads to an average exergy loss rate of $3.1 \mathrm{~kW}$ which is $4.5 \%$ higher than the uniformly packed thermal store (Figure 17c).

We also simulated case 3 which included five of the high void fraction regions modelled in case 2 equally spread around the base of the thermal store. This resulted in an exergy loss rate of $3.5 \mathrm{~kW}, 17.5 \%$ higher than the uniformly packed thermal store (Figure 17d).

Finally, we evaluate the case of a $2 \mathrm{~cm}$ wide region adjacent to all the walls of the store where the void fraction is increased to 0.75 . This non-uniformity in the void fraction results in significant disruption of the thermal front adjacent to the walls of the store (Figure 16). The disruption is smoothed out to some extent on each charge cycle but the net effect is an average exergy loss rate of $4.8 \mathrm{~kW}$. This is $63 \%$ higher than the uniformly packed thermal store (Figure $17 \mathrm{e})$.
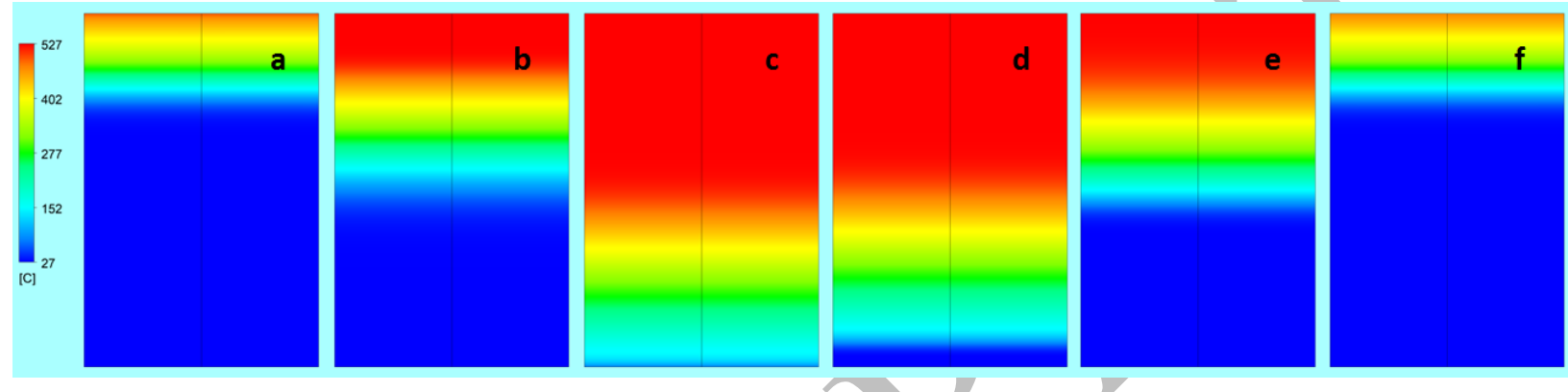

Figure 13 Contour plots of rock temperature during charge discharge cycle with void fraction case 2; each frame corresponds to a different time in the charge discharge cycle; $a=82000$ s (start of 5th charge cycle), $b=85000$, $c=92000 s, d=93000 s, e=98000 s, f=102000$ s (start of 5th discharge cycle)
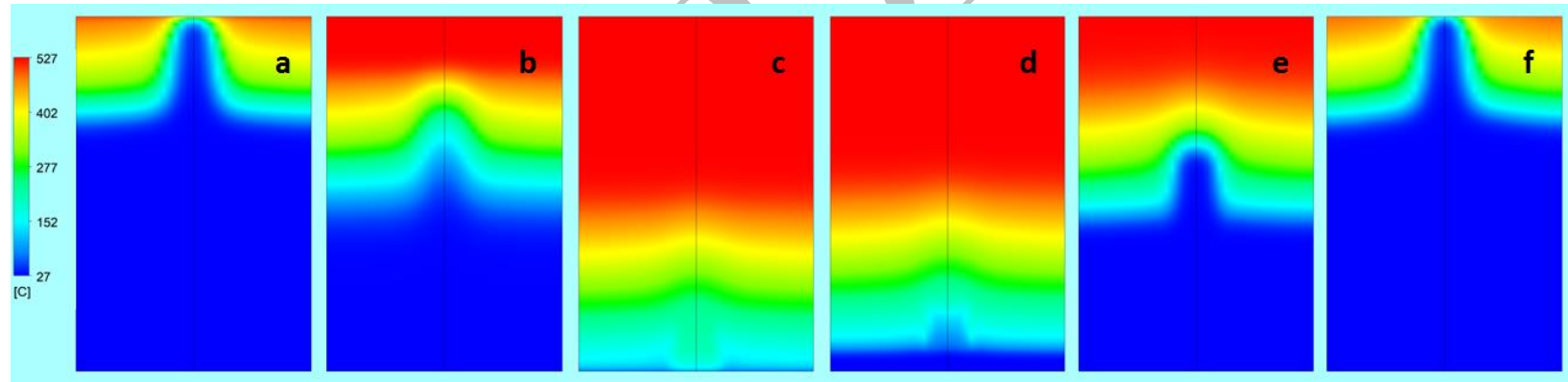

Figure 14 Contour plots of rock temperature during charge discharge cycle with void fraction case 2; each frame corresponds to a different time in the charge discharge cycle; $a=82000$ s (start of 5th charge cycle), $b=85000$ s, $c=92000 \mathrm{~s}, d=93000 \mathrm{~s}, e=98000 \mathrm{~s}, f=102000 \mathrm{~s}$ (end of 5 th charge cycle)

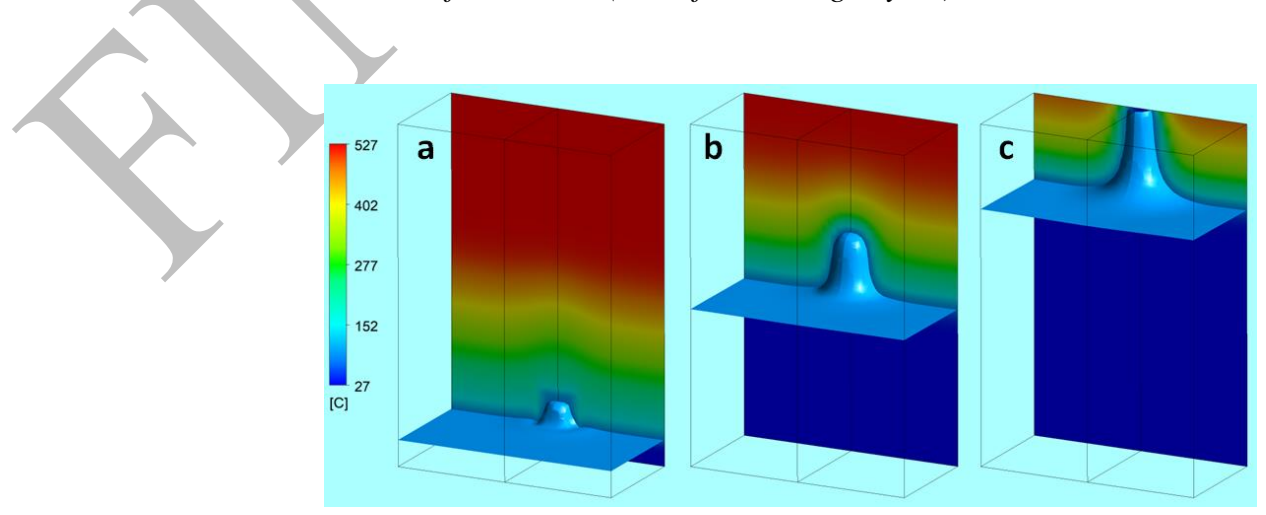

Figure $153 D$ visualisation of cold tunnel instability during 5th discharge cycle on thermal store with case 2 void fraction; $a=93000 s, b=98000 s, c=102000 s$ 

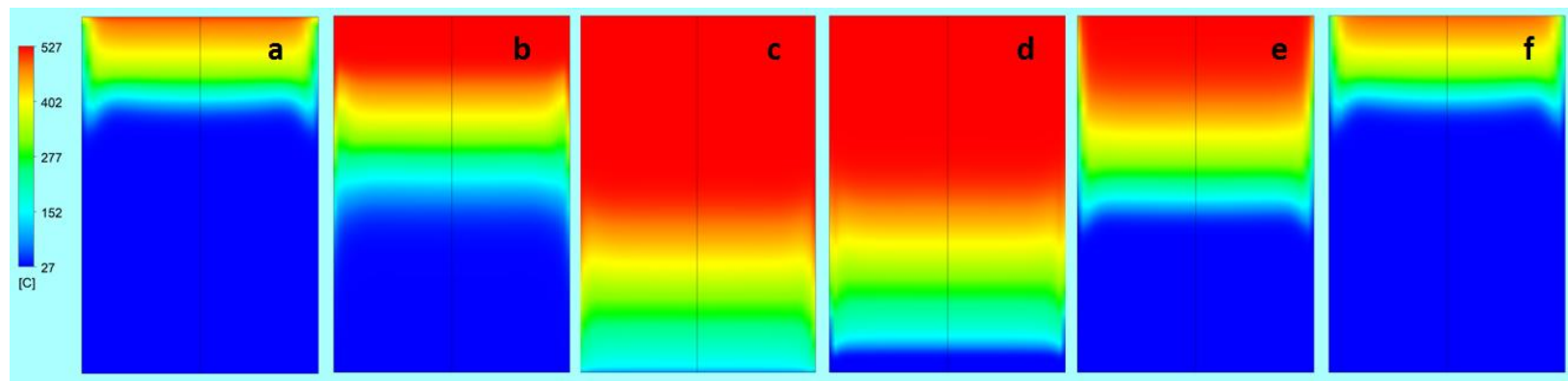

Figure 16 Contour plots of rock temperature during charge discharge cycle with void fraction case 4; each frame corresponds to a different time in the charge discharge cycle; $a=82000$ s (start of $5^{\text {th }}$ charge cycle), b=85000s, $c=92000 \mathrm{~s}, d=93000 \mathrm{~s}, e=98000 \mathrm{~s}, f=102000 \mathrm{~s}$ (end of $5^{\text {th }}$ discharge cycle)

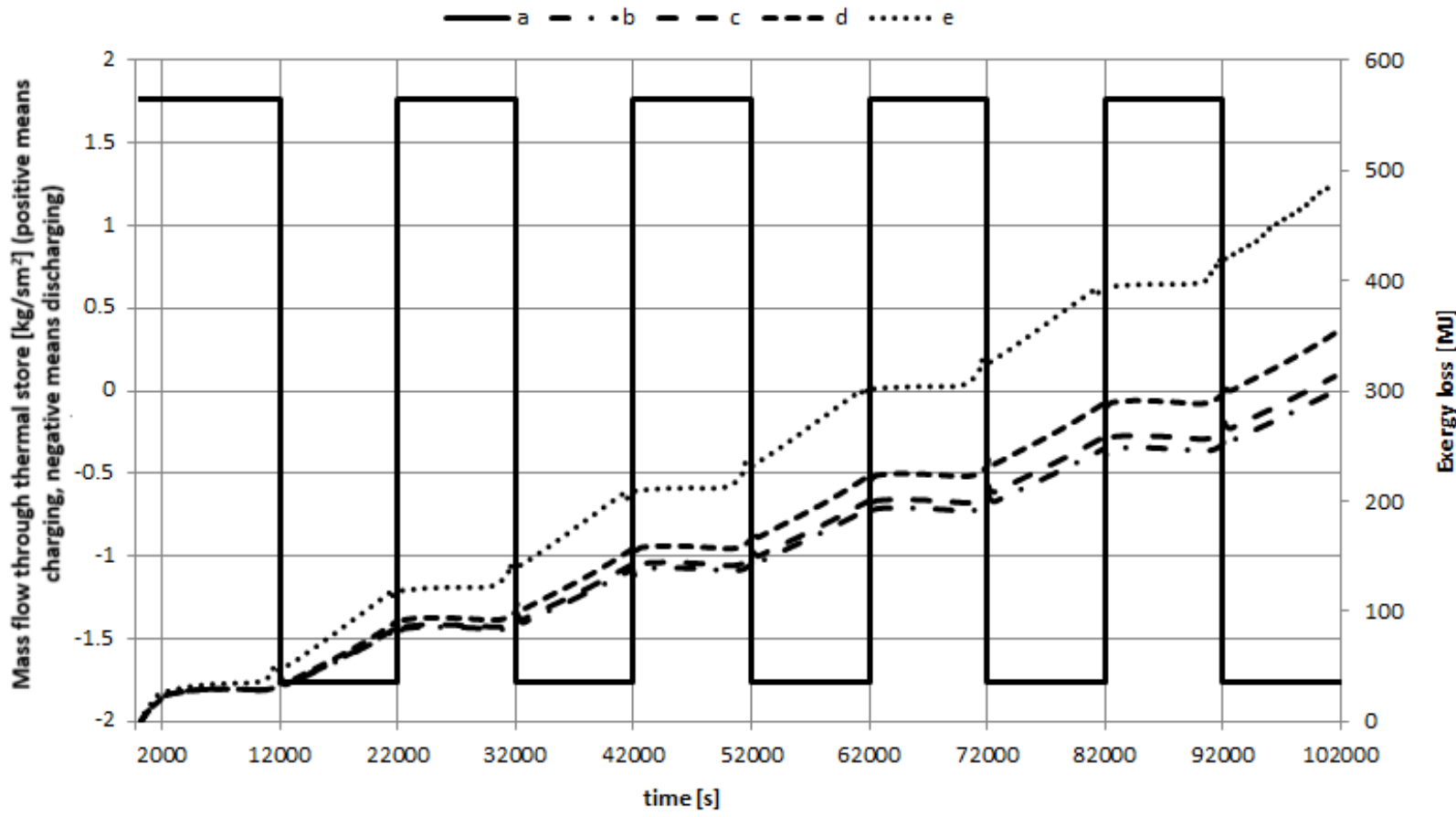

Figure 17 Exergy loss for example thermal store; $a$ is the mass flow through the thermal store during the chargeldischarge cycle; $b$ is the exergy loss for void fraction case 1; $c$ is the exergy loss for void fraction case 2; $d$ is the exergy loss for void fraction case 3; e is the exergy loss for void fraction case 4

\section{Discussion \& Conclusions}

Charging a packed bed thermal store with hot gas is found to be stable, while discharging it with cold gas is found to be inherently unstable. Any non-uniformities in inlet flow or void fraction in the packed bed can be the trigger for cold tunnelling during discharge of a hot thermal store. Equally this could be the case during charging of a cold store.

The stability of the thermal front depends on two factors:

1. the relationship between pressure gradient and temperature, and

2. transverse diffusion perpendicular to the main direction of flow through the packed bed.

When heating a packed bed both of these factors act to stabilise any perturbations in the thermal front. When cooling a packed bed, the fact that pressure drop is lower for preferentially cooled areas leads to further cooling in those areas. 
This positive feedback effect can result in significant perturbations of the thermal front. Transverse diffusion does work to spread out deformations of the thermal front during discharge, but it has been shown that unless the perturbation is smaller than a certain size (the order of 6-35cm) this effect does not appear to counter the inherent instability. It should also be noted that perturbations are more likely to develop in sharp edged thermoclines. The more thermoclines diffuse the less likely a perturbation in the thermal front will grow.

CFD simulations of a repeated charge/discharge cycle applied to an example packed bed, showed that a high void fraction region at inlet to the store resulted in an unstable perturbation of the thermal front that developed on every discharge cycle. This resulted in a $4.5 \%$ increase in the average exergy loss rate (as compared to a uniformly packed thermal store), due to the extra irreversible heat transfer occurring during discharge. Adding high void fraction adjacent to the store walls, which is hard to avoid when packing a store, was found to increase the average exergy loss rate by $63 \%$ as compared to the uniformly packed store.

While one dimensional models will give a good indication of thermal store losses, we suggest that for a truly accurate calculation of exergy loss three-dimensional modelling is required, including sensitivity analysis to regions of nonuniform void fraction. However, it should be noted that although 3D modelling can capture negative effects of variations in porosity and non-uniformities in flow, the magnitudes of these extra exergy losses do not appear to be significant enough to question the viability of packed bed thermal stores. Infact the results show how the performance of a thermal store is reasonably resilient to non-uniformities. In our case studies non uniformities in void fraction resulted in an averaged exergy loss rate between $1.6 \%$ and $2.5 \%$ of the rated exergy flow in and out of the store. For reference the exergy loss rate in the uniformly packed thermal store was $1.5 \%$ of the rated exergy flow.

In terms of mitigating potential exergy penalties due to 3D thermocline instabilities, three obvious techniques present themselves. Firstly, trying to ensure uniform void fraction, secondly promoting transverse thermal diffusion and finally ensuring the flow into the bottom of the thermal store is uniform. Keeping localised high void fraction regions below the critical perturbation size for the packed bed is the first line of defence as transverse diffusion will tend to dissipate perturbations smaller than this scale. One area where significant increase in void fraction is likely to occur over large areas is at the walls of a thermal store [30]. Adding a thin layer of flexible blanket insulation or foam inside the walls of the thermal store could mitigate this high void fraction region. One might also consider vibrating the packed bed as it is slowly filled to try to achieve more uniform packing. In terms of improving transverse diffusion, mixing high thermal conductivity waste metal swarf into the packed bed or adding conductive metallic meshes at regular intervals would be beneficial. Also using non-spherical packing material could act to increase effective transverse diffusion and thus help to stabilise thermal fronts when cooling. Achieving uniform inlet flows to the thermal store is best achieved by having a large manifold where the flow is decelerated as much as possible thus avoiding local regions where the flow could jet into the packed bed.

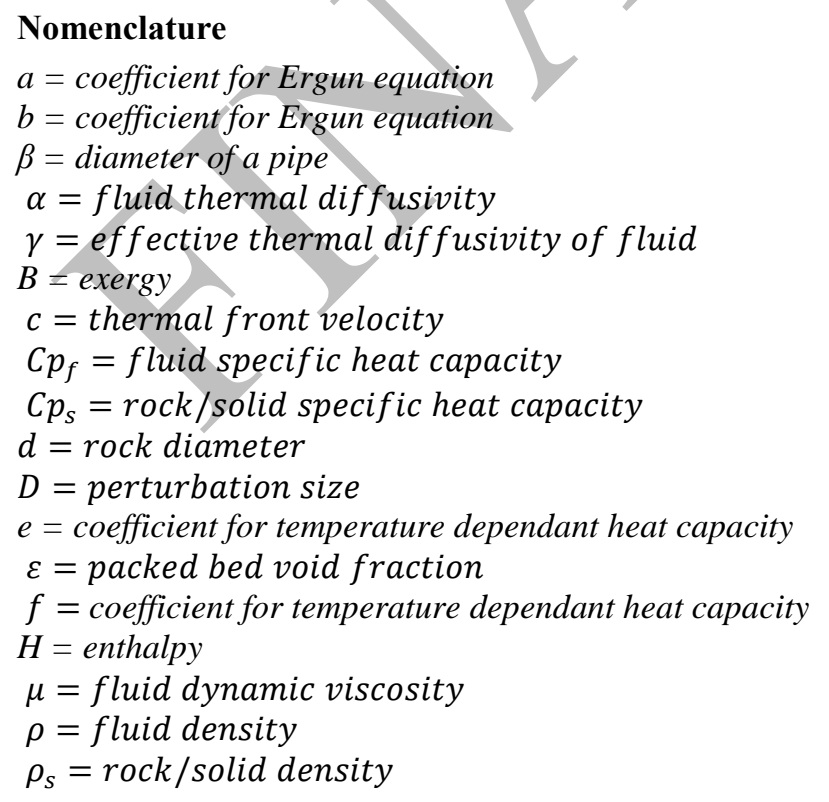


$k=$ constant relating thermal front velocity to mass flow rate per unit area

$k_{c}=$ effective fluid thermal conductivity

$k_{f}=$ fluid thermal conductivity

$k_{t}=$ effective transverse thermal conductivity

$K=$ area porosity tensor

$L=$ packed bed length or pipe length

$\Gamma=$ source term for heat transfer between solid and fluid

$M=$ mass flow rate per unit area

$\dot{m}=$ mass flow rate

$m=$ mass

$v=$ kinematic viscosity

$\Delta P=$ pressure drop

$P=$ pressure

$\mathrm{Pe}=$ Peclet number

$\sigma=$ flow resistance tensor

$Q=$ heat flow in or out of perturbation

$R=$ fill ratio

$S=$ cross sectional area of the bulk of the packed bed

$S_{c}=$ cross sectional area of the column control volume

$T=$ temperature

$\Delta T=$ temperature difference between top and bottom of thermal store

$U=$ velocity vector

$V=$ superficial velocity

$x=$ height of thermal front 


\section{References}

1. T Desrues, J Ruer, P Marty, JF Fourmigué "A thermal energy storage process for large scale electric applications" Applied Thermal Engineering 30(5):425-432 - April 2010

2. A. Smallbone, V. Jülchb , R. Wardlea \& A. P. Roskilly "Levelised Cost of Storage for Pumped Heat Energy Storage in comparison with other energy storage technologies" Energy Conversion and Management 152 221-228 Nov 2017

3. S.D.Garvey et al. "Analysis of a wind turbine power transmission system with intrinsic energy storage capability" Wind Engineering Volume 39 No 22015 pgs 149-174

4. E. Barbour et al. "Adiabatic Compressed Air Energy Storage with packed bed thermal energy storage" Applied Energy Volume 155, 1 October 2015, Pages 804-815

5. C._Bullough et al. "Advanced Adiabatic Compressed Air Energy Storage for the Integration of Wind Energy" Proceedings of the European Wind Energy Conference, EWEC 2004, $22-25$ November 2004, London UK

6. B. Cárdenas et al. "Lowering the cost of large-scale energy storage: High temperature adiabatic compressed air energy storage" Propulsion and Power Research. 2017

7. L. Geissbühler, V. Becattini, G. Zanganeh, S. Zavattoni, M. Barbato, A. Haselbacher, A. Steinfeld "Pilot-scale demonstration of advanced adiabatic compressed air energy storage, Part1: Plant description and tests with sensible thermal-energy storage” Energy Storage Mar 2018 129-139

8. T. Schumann, "Heat transfer: a liquid flowing through a porous prism" J. Franklin Inst. 208 (1929) 405-416.

9. M. Hanchen et al. "High-temperature thermal storage using a packed bed of rocks - Heat transfer analysis and experimental validation" Applied Thermal Engineering 31(10):1798-1806 · July 2011

10. S. Kuravi et al. "Investigation of a high temperature packed bed sensible heat thermal energy storage system with large sized elements" Proceedings of the ASME 2012 International Mechanical Engineering Congress \& Exposition IMECE2012 November 9-15, 2012, Houston, Texas, USA

11. M. Cascettaa et al. "A study of a packed-bed thermal energy storage device: test rig, experimental and numerical results" 69th Conference of the Italian Thermal Engineering Association, ATI 2014

12. White, A. J.: "Loss analysis of thermal reservoirs for electrical energy storage schemes", Appl. Energy, 2011, 88, 11, pp. $4150-4159$

13. White, A., McTigue, J., Markides, C. "Wave Propagation and Thermodynamic Losses in Packed-Bed Thermal Reservoirs for Energy,Storage” Applied Energy Volume 130, October 2014, Pages 648-657

14. Cascetta, M., Cau, G., Puddu, P., \& Serra, F. (2016). A comparison between CFD simulation and experimental investigation of a packed-bed thermal energy storage system. Applied Thermal Engineering, 98, 1263-1272.

15. Bruch, A., Fourmigue, J. F., Couturier, R., \& Molina, S. (2014). Experimental and numerical investigation of stability of packed bed thermal energy storage for CSP power plant. Energy Procedia, 49, 743-751.

16. Zavattoni, S. A., Barbato, M. C., Pedretti, A., \& Zanganeh, G. (2011, September). CFD simulations of a pebble bed thermal energy storage system accounting for porosity variations effects. In Solar PACES 2011 conference. Paper ID (Vol. 24636). 
17. Frank G.F. Qin, Xiaoping Yang, Zhan Ding, Yuanzhi Zuo, Youyan Shao, Runhua Jiang \& Xiaoxi Yang "Thermocline stability criterions in single-tanks of molten salt thermal energy storage" Applied Energy 97 (2012) $816-821$

18. B.S.Massey "Mechanics of Fluids" Chapman \& Hall; $6^{\text {th }}$ edition; 1989; ISBN: 978-0412342806

19. J. van Berkel, C.C.M. Rindt, A.A. van Steenhoven "Thermocline dynamics in a thermally stratified store" Heat and Mass Transfer 45 (2002) 343-356

20. A. Tinaikar, S. Advaith U. Kumar C.K.V. Manu \& S.Basu "Spatio-temporal disruption of thermocline by successive laminar vortex pairs in a single tank thermal energy storage" Applied Thermal Engineering Volume 109, Part B, 25 October 2016, Pages 924-935

21. Simone A. Zavattoni, Lukas Geissbühler, Maurizio C. Barbato, Giw Zanganeh, Andreas Haselbacher \& Aldo Steinfeld "High-Temperature Thermocline TES Combining Sensible and Latent Heat - CFD Modeling and Experimental Validation” AIP Conference Proceedings 1850, 080028 (2017)

22. S. Ergun, "Fluid flow through packed bed columns" Chem. Process engineering London 1952.

23. Y. Ming \& L. Jian-Hua "A fractal model for the transverse thermal dispersion conductivity in porous media" 2004 Chinese Phys. Lett. 21117

24. P.W.Dietz "Effective Thermal Conductivity of Packed Beds" Ind. Eng. Chem. Fundamen., 1979, 18 (3), pp 283286

25. B. Cardenas, T. Davenne, J. Rouse \& S.D. Garvey "Effect of Design Parameters on the Exergy Efficiency of a Utility-Scale Packed Bed” J. Energy Storage Aug 2018

26. ANSYS® Academic Research CFX, Release 17.1, ANSYS, Inc

27. I.Dincer \& M.A.Rosen "Exergy, Energy, Environment and Sustainable Development" 2nd edition 2013 Elsevier Science ISBN: 978-0-08-097089-9

28. P. Hartlieb M. Toifl, F. Kuchar, R. Meisels \& T. Antretter "Thermo-physical properties of selected hard rocks and their relation to microwave-assisted comminution" Minerals Engineering 91 34-41 (2016)

29. T. Davenne, S.D. Garvey, B. Cardenas \& M. Simpson "The cold store for a pumped thermal energy storage system" J. Energy Storage Mar 2017

30. L.H.S. Roblee, R.M. Baird \& J.W. Tierney "Radial porosity variations in packed beds" AIChE Journal Volume4, Issue4, Pages 460-46 December 1958 